\title{
SÍNTESE E AVALIAÇÃO DA ATIVIDADE ANTIMICROBIANA DE FURANONAS HALOGENADAS E DE COMPOSTOS ANÁLOGOS AOS NOSTOCLÍDEOS
}

\author{
Luiz C. A. Barbosa*, Célia R. A. Maltha, Antônio J. Demuner, Patrícia F. Pinheiro, Jodieh O. S. Varejão e Ricardo M. Montanari \\ Departamento de Química, Universidade Federal de Viçosa, Av. P. H. Rolfs, s/n, 36570-000 Viçosa - MG, Brasil \\ Nélio J. Andrade \\ Departamento de Ciência e Tecnologia de Alimentos, Universidade Federal de Viçosa, Av. P. H. Rolfs, s/n, 36570-000 Viçosa - MG, Brasil
}

Recebido em 22/2/10; aceito em 5/8/10; publicado na web em 25/10/10

\begin{abstract}
SYNTHESIS AND EVALUATION OF ANTIMICROBIAL ACTIVITY OF HALOGENATED FURANONES AND COMPOUNDS ANALOGUES TO NOSTOCLIDES. Considering the broad spectrum of biological activity of gamma-butyrolactone derivatives, we presented the synthesis of 3,4-dihalo-5-arylidenefuran-2(5H)-ones (17-21) and analogues (24-28) of the natural product nostoclide (7,8). Furanones 17-21 were synthesized from the condensation of aromatic aldehydes with lactones $\mathbf{1 4}$ and 15, that were obtained from mucobromic and mucochloric acids. Lactone $\mathbf{1 5}$ was converted into the intermediate $\mathbf{2 3}$ in $36 \%$ overall yield. Compound $\mathbf{2 3}$ was then transformed into the nostoclide analogues 24-28. Some of the compounds prepared showed antimicrobial activities against Escherichia coli, Staphylococcus aureus and Bacillus cereus comparable to commercial antibiotics.
\end{abstract}

Keywords: $\gamma$-alquilydenebutenolides; nostoclide derivatives; furanones.

\section{INTRODUÇÃO}

A utilização de compostos com atividades antimicrobianas gerou grande avanço em relação à prevenção e ao tratamento de doenças infecciosas. Entretanto, o uso generalizado dessas substâncias tem acarretado problemas diversos, principalmente o desenvolvimento de resistência dos micro-organismos aos antibióticos. ${ }^{1-3}$

No início dos anos 60 observou-se que a bactéria Staphylococcus aureus já era resistente à penicilina. Nas décadas de 70 e 80 surgiram novas bactérias Gram-positivas e Gram-negativas com diferentes mecanismos de resistência. Nos últimos anos tem-se observado o surgimento de micro-organismos altamente resistentes, quase sempre indestrutíveis ou com escassas opções terapêuticas para tratamento com os medicamentos disponíveis. ${ }^{4}$ Assim, há uma necessidade constante de descoberta de novos agentes antimicrobianos que possam ser empregados no tratamento de doenças diversas, causadas por micro-organismos resistentes às terapias convencionais utilizadas.

Diversos estudos estão sendo realizados para a busca de novas moléculas bioativas como alternativas terapêuticas. Compostos pertencentes a diferentes classes funcionais, como peptídeos, furanonas e terpenos, têm sido isolados de organismos marinhos (algas, esponjas, etc). Esses compostos apresentaram atividade biológica diversificada, sendo observada significativa atividade antimicrobiana na maioria dos casos. ${ }^{5}$

Dentre as furanonas bioativas que apresentam comprovada atividade antimicrobiana destacam-se as furanonas pertencentes à classe dos $\gamma$-alquilidenobutenolídeos (Figura 1). ${ }^{6-11}$ Diversos estudos mostraram que furanonas bromadas inibem a formação de biofilmes em várias espécies de bactérias. ${ }^{12}$ As substâncias conhecidas como fimbrolídeos (1), isoladas da alga vermelha Delisea pulchra, são inibidoras da formação de biofilmes em Pseudomonas aeruginosa, Escherichia coli, Bacillus subtilis, Staphylococcus epideridis e Streptococcus ssp. ${ }^{12-16}$ Ensaios realizados com compostos análogos aos fimbrolídeos (2) mostraram sua capacidade de inibição ao crescimento de diversas bactérias Gram-negativas e Gram-positivas. ${ }^{16-20}$

*e-mail: lcab@ufv.br

\#Artigo em homenagem ao Prof. Hans Viertler
Outras furanonas de ocorrência natural como a protoanemonina (3), ${ }^{21}$ o rubrolídeo A (4), ${ }^{22}$ o lissoclinolídeo $(5)^{23}$ e a pulvinona $(6)^{24}$ também apresentaram atividade antimicrobiana significativa.<smiles>[R]C(Br)=C1OC(=O)C(C([R])CCC)=C1Br</smiles><smiles>[R]C1=CC(=O)O/C1=C(/C)Br</smiles>

1 Fimbrolídeo $\mathrm{R}^{1}=\mathrm{H}, \mathrm{OH}, \mathrm{OAc}$ $\mathrm{R}^{2}=\mathrm{H}, \mathrm{Br}, \mathrm{Cl}, \mathrm{I}$

2 Fimbrolídeos $\mathrm{R}=\mathrm{H}, \mathrm{Br}$ sintéticos<smiles>O=C1C=C(c2cc(Br)c(O)c(Br)c2)/C(=C/c2cc(Br)c(O)c(Br)c2)O1</smiles>

3 Protoanemonina

4 Rubrolídeo A<smiles>O=C1O/C(=C/CCO)C=C1/C=C/CO</smiles>

5 Lissoclinolídeo<smiles>O=C1O/C(=C\c2ccccc2)C(O)=C1c1ccccc1</smiles>

6 Pulvinona
Figura 1. Estruturas de $\gamma$-alquilidenobutenolídeos com atividade antimicrobiana

Os nostoclídeos I (7) e II (8) (Figura 2) foram isolados da alga verde-azul Nostoc sp., encontrada no líquen Peltigera canina. ${ }^{25}$ Em 
função do interesse em avaliar as atividades biológicas desses compostos, foram desenvolvidas metodologias de síntese para os mesmos. ${ }^{26-28}$

Em continuidade ao nosso constante esforço na busca por novos compostos com atividades biológicas como antimalarial, ${ }^{29,30}$ anticâncer, ${ }^{31,32}$ inseticida, ${ }^{33,34}$ nematicida ${ }^{35,36}$ e fitotóxica ${ }^{31,37}$ foi realizada a síntese de alguns compostos análogos aos nostoclídeos e verificou-se que os mesmos apresentam atividade fitotóxica. ${ }^{38} \mathrm{Em}$ testes realizados com cloroplastos isolados de espinafre foi verificado que vários análogos aos nostoclídeos, incluindo 9 a 13 (Figura 2) inibem a fotossíntese, interferindo no transporte de elétrons dirigido pela luz, da água para o ferricianeto de potássio, um oxidante não biológico. ${ }^{31,39,40} \mathrm{~A}$ síntese desses compostos foi realizada tendo-se como objetivo inserí-los em um amplo programa de busca de novas substâncias com potencial atividade fitotóxica, que sejam seletivas, causem menor impacto ao meio ambiente e apresentem potencial desenvolvimento comercial.

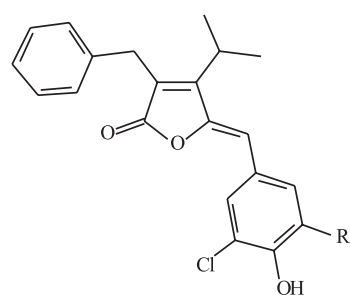

Nostoclídeo I $\quad \mathrm{R}=\mathrm{Cl} 7$ Nostoclídeo II $\mathrm{R}=\mathrm{H} \quad \mathbf{8}$

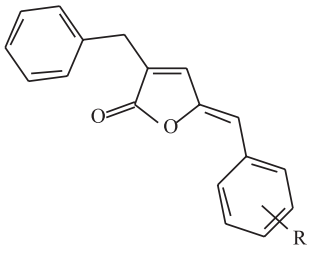

$\mathrm{R}=p-\mathrm{NO}_{2}$

$\mathrm{R}=m-\mathrm{NO}_{2} \quad \mathbf{1 0}$

$\mathrm{R}=p-\mathrm{CF}_{3} \quad 11$

$\mathrm{R}=m-\mathrm{CF}_{3} \quad 12$

$\mathrm{R}=p-\mathrm{C}_{2} \mathrm{H}_{5} \quad 13$
Figura 2. Estrutura de $\gamma$-alquilidenobutenolídeos (nostoclídeos e compostos análogos)

Algumas arilidenofuranonas análogas aos nostoclídeos também apresentaram citotoxicidade moderada em ensaios realizados com linhagens de células cancerígenas humanas HL-60 (leucemia), HCT-8 (câncer de cólon), MDA/MDB (câncer de mama) e SF295 (câncer do SNC). ${ }^{31}$

A atividade antibacteriana exibida por furanonas e $\gamma$-alquilidenobutenolídeos ${ }^{6-11,21-24}$ despertou o interesse para a investigação do potencial antimicrobiano de 3,4-dialo-5-benzilidenofuran-2( $5 H)$-onas e de análogos aos nostoclídeos. Nesse trabalho apresenta-se a síntese de 3,4-dialo-5-benzilidenofuran-2(5H)-onas 17 a 21 e de análogos aos nostoclídeos 24 a 28 . Estruturalmente, os compostos 24 a 28 correspondem aos análogos $\mathbf{9}$ a $\mathbf{1 3}$ acrescidos do grupo isopropila na posição $\beta$ do anel lactônico. Os ensaios para avaliação da atividade antibacteriana foram realizados pelo método de difusão em ágar, utilizando-se cepas das bactérias Escherichia coli, Staphylococus aureus e Bacilus Cereus. ${ }^{41}$

\section{PARTE EXPERIMENTAL}

\section{Procedimentos experimentais gerais}

Todas as reações foram realizadas sob atmosfera de nitrogênio. Os solventes tetra-hidrofurano (THF), éter dietílico, diclorometano e di-isopropiletilamina (DIPEA) foram purificados conforme procedimentos descritos na literatura. ${ }^{42}$ As soluções de brometo de isopropil magnésio e de brometo de benzilzinco utilizadas foram previamente preparadas. Os reagentes tetrakis(trifenilfosfina)paládio (0) $\left(\mathrm{Pd}\left(\mathrm{PPh}_{3}\right)_{4}\right)$, trifluorometanossulfonato de tert-butildimetilsilila (TBDMSOTf) e 1,8-diazobiciclo[5.4.0] undec-7-eno (DBU), disponíveis comercialmente, foram utilizados sem prévia purificação. Os espectros na região do infravermelho foram registrados em solução de diclorometano, em espectrômetro Perkin Elmer Spectrum 1000, na região de 4000 a $635 \mathrm{~cm}^{-1}$. Os espectros de RMN foram obtidos em espectrômetro Varian Mercury 300, utilizando-se $\mathrm{CDCl}_{3}$ como solvente e tetrametilsilano (TMS) como padrão interno de referência. Os espectros de massas foram obtidos em aparelho Shimadzu PQ5050A, operando por inserção direta, e com detector de ionização por impacto de elétrons de $70 \mathrm{eV}$. As temperaturas de fusão foram determinadas em aparelho MQAPF-301 e não foram corrigidas.

Para a purificação dos compostos utilizou-se a cromatografia em coluna de sílica gel 60 (70-230 mesh-ASTM Merck). Todas as reações foram monitoradas por cromatografia em camada delgada utilizando-se placas de sílica gel Camlab-Polygram Silk/UV ${ }_{254} \mathrm{com}$ $1,25 \mathrm{~mm}$ de espessura.

As furanonas halogenadas 14 e $\mathbf{1 5}$ foram sintetizadas a partir dos ácidos mucobrômico e mucoclórico, e obtidas com rendimentos de 80 e $78 \%$, respectivamente, conforme procedimento descrito na literatura. ${ }^{43,44}$

Os compostos análogos aos nostoclídeos (24-28) foram sintetizados conforme procedimento descrito para a síntese dos compostos 17 a 21, utilizando-se como material de partida a lactona 23.

\section{Procedimentos sintéticos}

5(Z)-3,4-dibromo-5-benzilidenofuran-2(5H)-ona (17)

A um balão bitubulado, sob atmosfera de nitrogênio, adicionou-se 3,4-dibromofuran-2(5H)-ona (14) (0,089 g; 0,370 mmol), diclorometano $(3 \mathrm{~mL})$, TBDMSOTf $(85 \mu \mathrm{L} ; 0,37 \mathrm{mmol})$, DIPEA $(155 \mu \mathrm{L}$; $0,60 \mathrm{mmol})$ e benzaldeído $(0,083 \mathrm{~g} ; 0,780 \mathrm{mmol})$. A mistura foi mantida sob agitação magnética à temperatura ambiente por $1 \mathrm{~h} \mathrm{e}$ submetida a refluxo. Em seguida, adicionou-se DBU (60 $\mu \mathrm{L} ; 0,63$ mmol) e a mistura reagente foi mantida sob refluxo por $1 \mathrm{~h}$. Logo após, foi realizada a extração da mistura com diclorometano (3 x 10 $\mathrm{mL})$ e a fase orgânica foi lavada com solução de $\mathrm{HCl} 3 \mathrm{~mol} \mathrm{~L}^{-1}(2 \mathrm{x}$ $15 \mathrm{~mL}$ ), solução saturada de $\mathrm{NaCl}(2 \times 15 \mathrm{~mL})$ e secada sobre $\mathrm{MgSO}_{4}$ anidro. $\mathrm{O}$ solvente foi removido sob pressão reduzida em evaporador rotativo resultando em material sólido marrom que, após purificação por cromatografia em coluna de sílica gel (hexano:diclorometano 2:1 v/v), forneceu um sólido. Esse material foi recristalizado em mistura hexano:diclorometano $(50: 1 \mathrm{v} / \mathrm{v})$ e resultou na obtenção de $18 \mathrm{mg}$ de um sólido marrom $(0,11 \mathrm{mmol}, 15 \%)$ da lactona 17. $\mathrm{T}_{\mathrm{f}}=100,0-100,6^{\circ} \mathrm{C}$. IV (filme $\mathrm{NaCl}, \mathrm{cm}^{-1}$ ) $\bar{v}_{\text {max }} 3057,1769,1647$, 1556, 1221, 1180, 990, 969, 869, 755, 696; RMN de ${ }^{1} \mathrm{H}(300 \mathrm{MHz}$, $\mathrm{CDCl}_{3}$ ) $\delta$ 6,16 (s, 1H, H-6); 7,39-7,43 (m, 3H, H-3'-H-5'); 7,79-7,82 (m, 2H; H-2'/H-6'); RMN de ${ }^{13} \mathrm{C}\left(75 \mathrm{MHz}, \mathrm{CDCl}_{3}\right) \delta 113,4$ (C-3); 114,5 (C-6); 129,3 (C-2'/C-6')*; 130,5 (C-4'); 131,3 (C-3'/C-5')*; 132,1 (C-1'); 137,6 (C-4); 145,8 (C-5); 163,6 (C-2). EM m/z (\%): $332\left(\mathrm{C}_{11} \mathrm{H}_{6} \mathrm{Br}_{2} \mathrm{O}_{2},[\mathrm{M}+4]^{+}, 48\right) ; 330\left([\mathrm{M}+2]^{+}, 100\right) ; 331\left([\mathrm{M}]^{+}, 12\right)$; 328 (49); 249 (11); 193 (39); 118 (65); 90 (83); 61 (70). *atribuições podem estar invertidas.

Os compostos 18 a 21 foram sintetizados empregando-se o mesmo procedimento descrito para a síntese de $\mathbf{1 7}$. Em todos os casos os compostos foram purificados por cromatografia em coluna de sílica gel utilizando-se como eluente hexano:diclorometano $2: 1 \mathrm{v} / \mathrm{v}$ e recristalizados em hexano:diclorometano 50:1 v/v.

5(Z)-3,4-dibromo-5-(3,4-metilenodioxibenzilideno)furan-2(5H)ona (18): sólido amarelo $(0,48 \mathrm{mmol}, 65 \%), \mathrm{T}_{\mathrm{f}}=170,5-171,2^{\circ} \mathrm{C}$; IV (filme $\left.\mathrm{NaCl}, \mathrm{cm}^{-1}\right) \bar{v}_{\text {max }} 3075,2917,1768,1651,1569,1502,1269$, 1254, 1106, 1017, 983, 930, 656. RMN de ${ }^{1} \mathrm{H}\left(300 \mathrm{MHz}, \mathrm{CDCl}_{3}\right) \delta$ : 6,04 (s, 2H, -O-C $\left.\underline{H}_{2}-\mathrm{O}-\right)$; 6,36 (s, $\left.1 \mathrm{H}, \mathrm{H}-6\right) ; 6,84$ (d, $1 \mathrm{H}, J=8,1 \mathrm{~Hz}$, $\mathrm{H}^{-5}$ '); 7,21 (dd, 1H, $J=8,1 \mathrm{~Hz}, J=1,8 \mathrm{~Hz}, \mathrm{H}-6^{\prime}$ ); 7,46 (d, 1H, $J=1,8$ $\mathrm{Hz}, \mathrm{H}-2$ '). RMN de ${ }^{13} \mathrm{C}\left(75 \mathrm{MHz}, \mathrm{CDCl}_{3}\right) \delta 101,8$ (-O- $\left.\underline{\mathrm{CH}}_{2}-\mathrm{O}-\right)$; 108,5 (C-5'); 109,0 (C-2'); 110,3 (C-6); 125,4 (C-6'); 127,5 (C-3); 129,9 (C-1'); 132,0 (C-4); 148,7 (C-3'); 149,8 (C-4'); 161,1 (C-2); 162,0 (C- 
5). $\mathrm{EM} \mathrm{m/z} \mathrm{( \% )} 376\left(\mathrm{C}_{12} \mathrm{H}_{6} \mathrm{Br}_{2} \mathrm{O}_{4},[\mathrm{M}+4]^{+}, 45\right) ; 374\left([\mathrm{M}+2]^{+}, 100\right)$; 372 ([M] $\left.{ }^{+}, 53\right) ; 162$ (52); 134 (58); 133 (24); 76 (82); 51 (27); 50 (86).

5(Z)-3,4-dibromo-5-(4-nitrobenzilideno)furan-2(5H)-ona (19): sólido amarelo $(0,08 \mathrm{mmol}, 13 \%), \mathrm{T}_{\mathrm{f}}=188,0-189,0{ }^{\circ} \mathrm{C}$. IV (filme $\left.\mathrm{NaCl}, \mathrm{cm}^{-1}\right) \bar{v}_{\text {max }} 3041,1724,1593,1609,1518,1345,1108,1024$, 859, 786. RMN de ${ }^{1} \mathrm{H}\left(300 \mathrm{MHz}, \mathrm{CDCl}_{3}\right) \delta 6,77$ (s, $\left.1 \mathrm{H}, \mathrm{H}-6\right) ; 8,00(\mathrm{~d}$, $2 \mathrm{H}, J=9 \mathrm{~Hz}, \mathrm{H}-3^{\prime} / \mathrm{H}-5$ '); 8,27 (d, 2H; $J=9 \mathrm{~Hz}, \mathrm{H}-2^{\prime} / \mathrm{H}-6$ '). RMN de ${ }^{13} \mathrm{C}\left(75 \mathrm{MHz}, \mathrm{CDCl}_{3}\right) \delta 105,0(\mathrm{C}-6) ; 119,1(\mathrm{C}-3) ; 123,8\left(\mathrm{C}-3^{\prime} / \mathrm{C}-5^{\prime}\right) *$; 129,8 (C-2'/C-6')*; 135,3 (C-4); 137,0 (C-1'); 146,0 (C-5); 148,5 (C-4'); 158,3 (C-2). EM m/z (\%) $377\left(\mathrm{C}_{11} \mathrm{H}_{5} \mathrm{Br}_{2} \mathrm{NO}_{4},[\mathrm{M}+4]^{+}, 45\right)$; $375\left([\mathrm{M}+2]^{+}, 100\right) ; 373\left([\mathrm{M}]^{+}, 53\right) ; 162(52) ; 134$ (58); 133 (24); 76 (82); 51 (27); 50 (86). *atribuições podem estar invertidas.

5(Z)-3,4-dicloro-5-(3,4-dioxametilbenzilideno)furan-2(5H)-ona (20): sólido amarelo $(0,83 \mathrm{mmol}, 70 \%), \mathrm{Tf}=159,0-160,0^{\circ} \mathrm{C}$. IV (filme $\left.\mathrm{NaCl}, \mathrm{cm}^{-1}\right) \bar{v}_{\text {max }} .3075,2916,1769,1651,1618,1570,1503,1453$, $1269,1254,1108,1015,803,741$. RMN de ${ }^{1} \mathrm{H}\left(300 \mathrm{MHz}, \mathrm{CDCl}_{3}\right) \delta$ 6,03 (s, 2H, -OC $\underline{H}_{2} \mathrm{O}-$ ); 6,31 (s, 1H, H-6); 6,84 (d, 1H, J = 8,1; H-5'); 7,18 (dd, $1 \mathrm{H}, J=8,1 ; J=1,8$; H-6'); 7,44 (d, 1H; $J=1,8$; H-2'). RMN de ${ }^{13} \mathrm{C}\left(75 \mathrm{MHz}, \mathrm{CDCl}_{3}\right) \delta 102,0\left(-\mathrm{OCH}_{2} \mathrm{O}-\right) ; 109,0$ (C-5'); 110,4 (C-2'); 113,0 (C-6); 126,2 (C-6'); 127,4 (C-3); 130,2 (C-1'); 132,3 (C-4); 148,7 (C-3'); 149,8 (C-4'); 161,5 (C-5); 162,4 (C-2). EM m/z, (\%) $288\left(\mathrm{C}_{12} \mathrm{H}_{6} \mathrm{Cl}_{2} \mathrm{O}_{4},[\mathrm{M}+4]^{+}, 11\right) ; 286\left([\mathrm{M}+2]^{+}, 65\right) ; 285([\mathrm{M}+$ $\left.1]^{+}, 21\right) ; 284\left([\mathrm{M}]^{+}, 100\right) ; 162$ (37); 51 (16); 50 (26).

5(Z)-3,4-dicloro-5-(4-nitrobenzilideno)furan-2(5H)-ona (21): sólido amarelo $(0,06 \mathrm{mmol}, 10 \%), \mathrm{T}_{\mathrm{f}}=150,0-150,5^{\circ} \mathrm{C}$. IV (filme $\left.\mathrm{NaCl}, \mathrm{cm}^{-1}\right) \bar{v}_{\text {max }}: 3060,2906,1771,1649,1625,1580,1510,1458$, $1275,1244,1110,1005,805,748$. RMN de ${ }^{1} \mathrm{H}\left(300 \mathrm{MHz}, \mathrm{CDCl}_{3}\right) \delta$ 6,44 (s, 1H; H-6); 7,92-7,95 (m, 2H, H-3'-H-5'); 8,26-8, 29 (m, 2H; H2'/H-6'). RMN de ${ }^{13} \mathrm{C}\left(75 \mathrm{MHz}, \mathrm{CDCl}_{3}\right) \delta 101,0$ (C-6), 121,7 (C-3), 122,9 (C-5'), 123,9 (C-3'), 126,9 (C-6'), 127,8 (C-2'), 141,0 (C-1'), 143,9 (C-5), 146,4 (C-4), 148,1 (C-4'), 160,0 (C-2). EM m/z (\%) 289 $\left(\mathrm{C}_{11} \mathrm{H}_{5} \mathrm{Cl}_{2} \mathrm{NO}_{4},[\mathrm{M}+4]^{+}, 12\right) ; 288\left([\mathrm{M}+3]^{+}, 9\right) ; 287\left([\mathrm{M}+2]^{+}, 67\right)$; $285\left([\mathrm{M}]^{+}, 100\right) ; 255$ (50); 163 (13); 133 (70); 51 (88); 50 (33).

3-bromo-4-isopropilfuran-2(5H)-ona (22): A um balão bitubulado, sob atmosfera de nitrogênio, adicionou-se magnésio metálico $(0,248 \mathrm{~g}$; 10,2 mmol), THF (10 mL) e solução de brometo de isopropila em THF anidro (0,836 g; 6,8 mmol, $10 \mathrm{~mL}$ de THF). A mistura foi mantida sob agitação magnética por $40 \mathrm{~min}$. Em outro balão bitubulado adicionou-se $\mathrm{CuBr}_{\mathrm{Me}} \mathrm{S}(0,349 ; 1,7 \mathrm{mmol})$ e THF $(25 \mathrm{~mL})$, sob atmosfera de nitrogênio a $-78^{\circ} \mathrm{C}$. Após 5 min sob vigorosa agitação magnética, essa mistura foi adicionada lentamente ao balão contendo reagente de Grignard, com o auxílio de seringa. Em seguida, uma solução de 3,4-dibromofuran2(5H)-ona (14) (0,411 g; $1,7 \mathrm{mmol})$ e $\mathrm{BF}_{3}$. $\mathrm{Et}_{2} \mathrm{O}(0,482 \mathrm{~g} ; 3,4 \mathrm{mmol})$, em $15 \mathrm{~mL}$ de THF anidro, foi adicionada ao balão, sob agitação magnética, mantendo-se a temperatura de $-78{ }^{\circ} \mathrm{C}$ por $1 \mathrm{~h}$ e temperatura ambiente por $15 \mathrm{~h}$. Solução saturada de cloreto de amônio $(50 \mathrm{~mL})$ foi adicionada à mistura, que permaneceu sob agitação magnética por $30 \mathrm{~min}$. A fase aquosa extraída com éter dietílico $(3 \times 50 \mathrm{~mL})$ e a fase orgânica concentrada em evaporador rotatório, secada sobre $\mathrm{MgSO}_{4}$ anidro e filtrada e concentrada em evaporador rotatório. A lactona $\mathbf{2 2}$ foi obtida como um óleo de coloração amarelada, com rendimento de 93\% (0,324 g, 1,6 $\mathrm{mmol}$ ), determinado pelo método do padrão interno utilizando a técnica de cromatografia gasosa. IV (filme $\mathrm{NaCl}, \mathrm{cm}^{-1}$ ) $v^{-}$: 2969, 2931, 2873, $1773,1637,1458,1015,985,755,724 . \mathrm{RMN}$ de ${ }^{1} \mathrm{H}\left(300 \mathrm{MHz}, \mathrm{CDCl}_{3}\right)$ $\delta 1,20$ (d, 6H, $J=7 \mathrm{~Hz}, \mathrm{H}-2$ '); 3,10 (sept, 1H, $J=7 \mathrm{~Hz}, \mathrm{H}-1$ ' ); 4,80 (s, $2 \mathrm{H}, \mathrm{H}-5)$. RMN de ${ }^{13} \mathrm{C}\left(75 \mathrm{MHz}, \mathrm{CDCl}_{3}\right.$ ) $\delta 20,1$ (C-2'); 29,1 (C-1'); 70,7

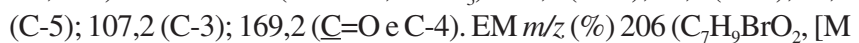
$\left.+2]^{+} ; 6\right) ; 204\left([\mathrm{M}]^{+}, 6\right) ; 164$ (27); 162 (26); 125 (19); 97 (17); 67 (53); 65 (26); 51 (28); 43 (100).
3-benzil-4-isopropilfuran-2(5H)-ona (23): Em um balão bitubulado adicionou-se zinco (1,178 g; $18 \mathrm{mmol})$ e $1 \mathrm{~mL}$ de THF anidro. A mistura foi sonicada por 15 min, seguida de agitação magnética por $5 \mathrm{~min}$, à temperatura ambiente. Na sequência foi adicionada solução de brometo de benzila $(0,71 \mathrm{~mL} ; 6 \mathrm{mmol})$ em THF $(3 \mathrm{~mL})$ e a mistura foi aquecida sob refluxo por $40 \mathrm{~min}$. Após o consumo total do brometo de benzila, foi adicionado THF anidro $(8 \mathrm{~mL})$. Em outro balão bitubulado, sob atmosfera de nitrogênio, adicionouse 3-bromo-4-isopropilfuran-2(5H)-ona (22) (1,03 g; 5,0 mmol), $\mathrm{Pd}\left(\mathrm{PPh}_{3}\right)_{4}(0,290 \mathrm{~g} ; 0,25 \mathrm{mmol})$, dimetilformamida $(25 \mathrm{~mL})$ e THF (13 mL). A mistura foi aquecida a $60^{\circ} \mathrm{C}$, sob agitação magnética. Após 10 min sob refluxo adicionou-se lentamente solução de brometo de benzilzinco previamente preparada $\left(0,5 \mathrm{~mol} \mathrm{~L}^{-1} ; 12 \mathrm{~mL} ; 6,0 \mathrm{mmol}\right)$, e o refluxo foi mantido por $5 \mathrm{~h}$. Solução saturada de cloreto de amônio (200 mL) e a extração realizada com acetato de etila $(5$ x $50 \mathrm{~mL})$. A fase orgânica foi lavada com água destilada $(6$ x $50 \mathrm{~mL})$, secada com $\mathrm{MgSO}_{4}$ anidro e concentrada em evaporador rotatório, fornecendo um sólido marrom que foi purificado por cromatografia em coluna de sílica gel (diclorometano:hexano 3:1 v/v + 1\% acetato de etila). A lactona $\mathbf{2 3}$ foi obtida como um sólido amarelo com rendimento de $36 \%\left(0,390 \mathrm{~g} ; 1,8 \mathrm{mmol}\right.$ ). IV (filme $\left.\mathrm{NaCl}, \mathrm{cm}^{-1}\right) v^{-}{ }_{\text {max }} 3061,3027$, 2963, 2921, 2850, 1750, 1665, 1601, 1494, 1453, 1345, 1173, 1070; 1055, 1010, 699. RMN de ${ }^{1} \mathrm{H}\left(300 \mathrm{MHz}, \mathrm{CDCl}_{3}\right) \delta: 1,11$ (d, $6 \mathrm{H}, J$ = 7, H-2"); 3,07 (sept., 1H, J = 7, H-1"); 3,62 (s, 2H, H-6); 4,72 (s, $2 \mathrm{H}, \mathrm{H}-5) ; 7,25$ (m, 5H, H-2'-H-6'). RMN de ${ }^{13} \mathrm{C}\left(75 \mathrm{MHz}, \mathrm{CDCl}_{3}\right) \delta$ 21,2 (C-2"'); 27,5 (C-1"'); 29,7 (C-6); 69,0 (C-5); 126,7 (C-4'); 128,7 (C-3'/C-5'); 128,8 (C-3); 128,8 (C-2'/C-6'); 138,5 (C-1'); 167,1 (C-4); 175,3 (C-2). EM m/z (\%) $216\left(\mathrm{C}_{14} \mathrm{H}_{16} \mathrm{O}_{2}\right)(10) ; 173$ (25); 155 (10); 129 (100); 91 (37); 65 (22); 43 (20).

5(Z)-3-benzil-4-isopropil-5-(4-nitrobenzilideno)furan-2(5H)-ona (24): sólido amarelo; $\mathrm{T}_{\mathrm{f}}=89,8-90,7^{\circ} \mathrm{C}$. IV (filme $\mathrm{NaCl}, \mathrm{cm}^{-1}$ ) $v^{-}{ }_{\max }$ 3100, 3070, 2925, 2852, 1768, 1518, 1433, 1342, 1110, 1075, 737, 695. RMN de ${ }^{1} \mathrm{H}\left(300 \mathrm{MHz}, \mathrm{CDCl}_{3}\right) \delta 1,34$ (d, 6H, $J=7 \mathrm{~Hz}, \mathrm{H}-2$ "); 3,19 (sept, $1 \mathrm{H}, J=7 \mathrm{~Hz}, \mathrm{H}-1$ ") ) 3,83 (simpleto largo, 2H, H-7); 6,20 (s, 1H, H-6); 7,20-7,33 (m, 5H, , H-2'-H-6'); 7,92 (d, 2H, J=9 Hz, H-2"'/H-6"'); 8,22 (d, 2H, J=9 Hz, H-3"'/H-5'"). RMN de ${ }^{13} \mathrm{C}(75$ $\mathrm{MHz}, \mathrm{CDCl}_{3}$ ) $\delta 21,8(\mathrm{C}-2$ "); 26,8 (C-1" ); 30,0 (C-7); 107,7(C-6); 123,1 (C-3"'/C-5'”); 127,0 (C-4'); 128,6 (C-3'/C-5' '); 129,0 (C-2'/C-6')*; 131,1 (C-2"'/C-6"'); 135,0 (C-1"”); 137,8 (C-1'); 139,2 (C-3); 139,9 (C-4"'); 149,9 (C-4); 157,6 (C-5); 170,0 (C-2). EM m/z (\%) 349 ([M]+, $\left.\mathrm{C}_{21} \mathrm{H}_{19} \mathrm{NO}_{4}, 79\right) ; 334$ (8); 321 (27); 306 (23); 260 (7); 215 (18); 152 (23); 141 (21); 128 (30); 115 (32); 105 (17); 91 (100); 84 (66); 65 (24). NOEDIFF (300 MHz, $\mathrm{CDCl}_{3}$ ): irradiação em $\delta 6,20(\mathrm{H}-6)$ causou incremento nos sinais em $\delta 1,34$ (7,72\%, H-2"'); 3, 19 (12,31\%, H-1") e 7,92 (17,54\%, H-2"'/H-6"'). *atribuições podem estar trocadas.

5(Z)-3-benzil-4-isopropil-5-(3-nitrobenzilideno)furan-2(5H)ona (25): sólido amarelo, $\mathrm{T}_{\mathrm{f}}=124,5-125,2^{\circ} \mathrm{C}$. IV (filme $\mathrm{NaCl}, \mathrm{cm}^{-1}$ ) $v^{-}{ }_{\max } 3100,3078,3038,1766,1645,1614,1530,1495,1350,1099$, $1033,1005,910,726,700,677 . \mathrm{RMN}$ de ${ }^{1} \mathrm{H}\left(300 \mathrm{MHz}, \mathrm{CDCl}_{3}\right) \delta$ 1,34 (d, 6H, $J=7,0 \mathrm{~Hz}, \mathrm{H}-2$ "'); 3,19 (sept, $1 \mathrm{H}, J=7,0 \mathrm{~Hz}, \mathrm{H}-1$ "'); 3,83 (s, 2H, H-7); 6,20 (s, 1H, H-6); 7,20-7,32 (m, 5H, H-2'-H-6'); 7,57 (t, $1 \mathrm{H}, J=8,0 \mathrm{~Hz}, \mathrm{H}-5$ "' ) ; 8,13 (ddd, $1 \mathrm{H}, J=2,1 \mathrm{~Hz}, J=0,9$ $\mathrm{Hz}, J=8,0 \mathrm{~Hz}, \mathrm{H}-4$ '"'); 8, 17 (dupleto largo, $1 \mathrm{H}, J=8,0 \mathrm{~Hz}, \mathrm{H}-6$ '”'); $8,44$ (t, $1 \mathrm{H}, J=2,1 \mathrm{~Hz}, \mathrm{H}-2$ "' $)$. RMN de ${ }^{13} \mathrm{C}\left(75 \mathrm{MHz}, \mathrm{CDCl}_{3}\right) \delta$ 21,8 (C-2"); 26,8 (C-1"'); 29,9 (C-7); 107,7 (C-6); 123,1 (C-4"'); 125, 1 (C-6'”'); 126,9 (C-4'); 128,5 (C-3'/C-5' '*; 129,0 (C-2'/C-6')*; 130,0 (C-3"' ); 135,1 (C-1'”); 136,0 (C-3); 138,0 (C-2'”'); 136,7 (C1'); 139,1 (C-4); 157,6 (C-5); 149,2 (C-5”); 170,0 (C-2). EM m/z (\%): $349\left([\mathrm{M}]^{+}, \mathrm{C}_{21} \mathrm{H}_{19} \mathrm{NO}_{4}, 45\right) ; 332(17) ; 306(7) ; 266(5) ; 215$ (10); 141 (14); 128 (17); 115 (21); 91 (100); 89 (23). *atribuições podem estar trocadas. 
5(Z)-3-benzil-4-isopropil-5-(4-trifluorometilbenzilideno)furan2(5H)-ona (26): sólido branco; $\mathrm{T}_{\mathrm{f}}=83,3-83,6^{\circ} \mathrm{C}$. IV (filme $\mathrm{NaCl}$, $\left.\mathrm{cm}^{-1}\right) v_{\text {max }}^{-} 3029,2972,2932,1769,1649,1615,1495,1416,1324$, $1165,1124,1069,964,699$. RMN de ${ }^{1} \mathrm{H}\left(300 \mathrm{MHz} \mathrm{CDCl}_{3}\right) \delta 1,33$ (d, 6H, $J=7,2 \mathrm{~Hz}, \mathrm{H}-2$ "); 3, 17 (sept, 1H, $J=7,2 \mathrm{~Hz}, \mathrm{H}-1$ "); 3,83 (s, 2H, H-7); 6,20 (s, 1H, H-6); 7,20-7,33 (m, 5H, H-2'-H-6' ); 7,62 (d, $2 \mathrm{H}, J=8,4 \mathrm{~Hz}, \mathrm{H}-3$ "' /H-5"'); 7,88 (d, 2H, $J=8,4 \mathrm{~Hz}, \mathrm{H}-2$ '”/H-6'"). $\mathrm{RMN} \mathrm{de}{ }^{13} \mathrm{C}\left(75 \mathrm{MHz}, \mathrm{CDCl}_{3}\right) \delta 21,8(\mathrm{C}-2$ ") $)$; 26,8 (C-1" ); 29,9 (C7); 108,7 (C-6); 125,8 (q, $J_{C, F}=3,7 \mathrm{~Hz}, \mathrm{C}-3$ "' $/ \mathrm{C}-5$ "' $)$; 126,0 $\left(\mathrm{CF}_{3}\right)$; 126,9 (C4'), 128,5 (C-2'/C-6'); 128,9 (C-3'/C-5'); 130,6 (C-4”'); 130,7 (C-2'"'/C-6"'); 134,0 (C-3); 136,5 (C-1'"'); 136,8 (C-1'); 138,0 (C-4); 157,7 (C-5); 170,3 (C-2). EM m/z (\%) $372\left([\mathrm{M}]^{+}, \mathrm{C}_{22} \mathrm{H}_{19} \mathrm{~F}_{3} \mathrm{O}_{2}\right.$, 100); 329 (29); 301 (15); 215 (15); 173 (14); 158 (32); 141 (22); 115 (35); 105 (17); 91 (100); 65 (25); 51 (14).

5(Z)-3-benzil-4-isopropil-5-(3-trifluorometilbenzilideno)furan2(5H)-ona (27): sólido branco; $\mathrm{T}_{\mathrm{f}}=89,9-90,8{ }^{\circ} \mathrm{C}$. IV (filme $\mathrm{NaCl}$, $\left.\mathrm{cm}^{-1}\right) \bar{v}_{\max } 3090,3060,2971,2932,1775,1613,1500,1464,1329$, $1167,1127,1075,1002,987,802,696,655$. RMN de ${ }^{1} \mathrm{H}(300 \mathrm{MHz}$, $\mathrm{CDCl}_{3}$ ) $\delta 1,33$ (d, $6 \mathrm{H}, J=7,2 \mathrm{~Hz}, \mathrm{H}-2$ ") ) 3,17 (sept, $1 \mathrm{H}, J=7,2 \mathrm{~Hz}$, H-1"); 3,82 (simpleto largo, 2H, H-7); 6,20 (s, 1H, H-6); 7,19-7,32 (m, 5H, H-2'-H-6'); 7,48-7,56 (m, 2H, H-5'" e H-4'"); 7,88 (simpleto largo, $1 \mathrm{H}, \mathrm{H}-2$ "'); 8,08 (dupleto largo, $1 \mathrm{H}, J=6,9 \mathrm{~Hz}, \mathrm{H}-6$ '"). RMN de ${ }^{13} \mathrm{C}\left(75 \mathrm{MHz}, \mathrm{CDCl}_{3}\right) \delta$ 21,8 (C-2"'); 25,9 (C-1"); 29,9 (C-7); 108,8 (C-6); 125,0 (q, $J_{C, F}=3,6 \mathrm{~Hz}, \mathrm{C}-4$ '”); $125,2\left(\mathrm{CF}_{3}\right) ; 126,8$ (q, $J_{C, F}=$ 3,8 Hz, C-2'"'); 127,1 (C-4'); 128,5 (C-2'/C-6'); 128,9 (C-3'/C-5'); 129,5 (C-5'”); 130,6 (C-3"'); 133,2 (C-6"'); 133,8 (C-3); 133,9(C1'”); 136,9 (C-1'); 139,33 (C-4); 148,46 (C-5); 169,88 (C-2). EM $m / z(\%): 372\left([\mathrm{M}]^{+}, \mathrm{C}_{22} \mathrm{H}_{19} \mathrm{~F}_{3} \mathrm{O}_{2}, 30\right) ; 344$ (8); 330 (100); 289 (29); 155 (28); 115 (16); 91 (50); 84 (30); 65 (12); 51 (17).

5(Z)-3-benzil-4-isopropil-5-(4-etilbenzilideno)furan-2(5H)-ona (28): sólido branco; $\mathrm{T}_{\mathrm{f}}=87,2-88,4^{\circ} \mathrm{C}$. IV (filme $\mathrm{NaCl}, \mathrm{cm}^{-1}$ ) $\bar{v}_{\text {max }} 3061$, 3027, 2927, 2852, 1755, 1650, 1604, 1494, 1454, 1109, 1039, 863, 699. $\mathrm{RMN}$ de ${ }^{1} \mathrm{H}\left(300 \mathrm{MHz}, \mathrm{CDCl}_{3}\right) \delta 1,24\left(\mathrm{t}, 3 \mathrm{H}, J=7,5 \mathrm{~Hz}, \mathrm{CH}_{2} \mathrm{CH}_{3}\right)$; 1,31 (d, $6 \mathrm{H}, J=7,2 \mathrm{~Hz}, \mathrm{H}-2$ "); 2,65 (q, $2 \mathrm{H}, J=7,2 \mathrm{~Hz}, \mathrm{CH}_{2} \mathrm{CH}_{3}$ ); 3,15 (sept, 1H, $J=7,2 \mathrm{~Hz}, \mathrm{H}-1$ "); 3,81 (s, 2H, H-7); 6,16 (s, 1H, H-6); 7,20 (d, 2H, $J=8,1 \mathrm{~Hz}, \mathrm{H}-3$ '”/H-5'"'); 7,25-7,31 (m, 5H, H-2'-H-6'); 7,66 (d, $2 \mathrm{H}, J=8,1 \mathrm{~Hz}, \mathrm{H}-2$ '"'/H-6"'). RMN de ${ }^{13} \mathrm{C}\left(75 \mathrm{MHz}^{\mathrm{CDCl}} \mathrm{CDC}_{3}\right) \delta 15,6$ $\left(\mathrm{CH}_{2} \mathrm{CH}_{3}\right) ; 21,9$ (C-2"); 26,7 (C-1"); 29,0 ( $\left.\mathrm{CH}_{2} \mathrm{CH}_{3}\right) ; 29,8$ (C-7); 110,8 (C-6); 126,7 (C-4'); 128,5 (C-3"'/C-5'"'); 128,6 (C-3'/C-5'); 128,9 (C-2'/C-6')"; 129,1 (C-2'”/C-6"'); 130,8 (C-1'”); 130,9 (C-3); 138,5 (C-1'); 145,6 (C-4); 146,8 (C-4”"); 158,0 (C-5); 171,0 (C-2). EM m/z (\%) $332\left([\mathrm{M}]^{+}, \mathrm{C}_{23} \mathrm{H}_{24} \mathrm{O}_{2}, 100\right) ; 317$ (16); 261 (8); 241 (6); 211 (12); 183 (9); 146 (12); 131 (42); 115 (21); 91 (50); 84 (28); 65 (9); 51 (11).

\section{Ensaios biológicos}

\section{Avaliação da atividade antibacteriana}

Para a avaliação da atividade antimicrobiana foram utilizadas as bactérias Gram-positivas Staphylococcus aureus (ATCC 25923) e Bacillus cereus, Ribotipo $1222-173-$ S4, isoladas de superfícies de equipamentos de pós-pasteurização ${ }^{45}$ e a Gram-negativa Escherichia coli. ${ }^{46}$

Foi empregado o método de difusão em disco em meio sólido, de acordo com as normas do National Committee for Clinical Laboratory Standards (NCCLS, 2003), atualmente nomeado Clinical and Laboratory Standards Institute (CLSI).

Placas de Petri contendo ágar Müeller-Hinton foram inoculadas com suspensões das bactérias utilizadas $\left(2 \times 10^{8} \mathrm{UFC} \mathrm{mL}^{-1}\right)$. Os compostos foram dissolvidos em dimetilsulfóxido $\left(20 \mathrm{mg} \mathrm{mL}^{-1}\right)$. Discos de papel de filtro (6 mm de diâmetro) foram embebidos com $10 \mu \mathrm{L}$ da solução teste de cada composto avaliado e posicionados sobre a superfície do ágar das placas inoculadas. As placas foram incubadas por $48 \mathrm{~h}$ a $35^{\circ} \mathrm{C}$. Os diâmetros das zonas de inibição foram medidos com paquímetro e expressados em milímetros. Os antibióticos vancomicina (30 $\mu \mathrm{g} / \mathrm{disco})$, penicillina G (10 UI), eritromicina (15 $\mu \mathrm{g} /$ disco $)$, gentamicina (10 $\mu \mathrm{g} / \mathrm{disco})$ e estreptomicina ( $10 \mu \mathrm{g} / \mathrm{disco})$ foram utilizados como controles positivos e água esterilizada e DMSO como controles negativos. Cada composto foi testado em triplicata e repetido três vezes, totalizando nove repetições. Os experimentos foram montados em delineamento inteiramente casualizado e os resultados foram analisados pela ANOVA e teste de Tukey a $\mathrm{P} \leq 0,05$, utilizando o programa computacional GENES (Genetics and Statistical Analysis Versão 2007.0.0 - Universidade Federal de Viçosa).

\section{RESULTADOS E DISCUSSÃO}

\section{Síntese de 3,4-di-halo-5-arilidenofuran-2(5H)-ona}

Utilizando procedimento da literatura, ${ }^{43,44}$ os ácidos mucobrônico e mucoclórico, disponíveis comercialmente, foram convertidos em 3,4-dibromofuran-2(5H)-ona (14) (75\%) e 3,4-diclorofuran-2(5H)ona (15) $(60 \%)$.

Posteriormente, a reação com diferentes aldeídos aromáticos, sob condições de reação de alquilidenação, ${ }^{47}$ forneceu as correspondentes 3,4-di-halo-5-arilidenofuran-2(5H)-onas $\mathbf{1 7}$ a 21, cujos rendimentos estão apresentados no Esquema 1. Não há na literatura, até o momento, relatos da síntese desses compostos, exceto para o caso de 21, que foi obtido pela desidratação do derivado aldólico correspondente. ${ }^{48}$

A reação de alquilidenação envolve uma etapa de eliminação $\beta$ e pode ser aplicada à síntese de vários $\gamma$-alquilidenobutenolídeos naturais e sintéticos. ${ }^{26}$ Inicialmente ocorre a formação in situ dos sililfuranos 14a e 15a, conforme apresentado no Esquema 1. A reação de adição entre esses furanos e diferentes aldeídos aromáticos, na presença de di-isopropiletilamina (DIPEA) e excesso do ácido de Lewis trifluorometanossulfonato de tert-butildimetilsilila (TBDMSOTf), resultou na formação dos intermediários aldólicos correspondentes (16a e 16b), não isolados. A eliminação do grupo tert-butildimetilsiloxila a partir desses adutos ocorreu empregando-se 1,8-diazabiciclo[5.4.0]undec-7eno (DBU) sob refluxo, e subsequente adição de $\mathrm{HCl} 3 \mathrm{~mol} \mathrm{~L}^{-1}$.

Essa reação é altamente estereosseletiva, resultando na formação preferencial ou exclusiva do estereoisômero com configuração Z, em concordância com a literatura. ${ }^{49,50}$ Os dados espectroscópicos obtidos para os compostos $\mathbf{1 7}$ a $\mathbf{2 1}$ confirmaram a formação exclusiva do isômero $Z$. A presença do átomo de halogênio na posição $\beta$ do anel lactônico representa um fator estrutural determinante para a estereosseletividade na obtenção desses compostos. Dados de experimentos bidimensionais de RMN (HSQC e HMBC) confirmaram as atribuições para os sinais em RMN de ${ }^{1} \mathrm{H}$ e de ${ }^{13} \mathrm{C}$.

Pelos dados do Esquema 1 observa-se que os rendimentos da reação de alquilidenação são melhores nos casos em que o anel benzilideno apresenta grupos doadores de elétrons. Embora três dos produtos tenham sido obtidos com rendimentos muito baixos, nenhum esforço foi feito para otimizar esta reação, uma vez que nesta etapa do trabalho o interesse nesses compostos estava direcionado para a avaliação preliminar de suas atividades antimicrobianas.

\section{Síntese dos compostos análogos aos nostoclídeos}

A síntese dos compostos $\mathbf{2 4}$ a $\mathbf{2 8}$ foi planejada de modo a manter na estrutura dos análogos os grupos benzila e isopropila no anel lactônico, presentes nos nostoclídeos I e II, e introduzir diferentes substituintes no anel aromático do grupo arilideno, de modo a avaliar a influência desses substituintes sobre a atividade biológica.

$\mathrm{O}$ procedimento original descrito para a síntese de $\mathbf{2 2}$ a partir de $\mathbf{1 4}^{27}$ envolve o uso de reagentes organoestânicos altamente tó- 
<smiles>[X]C1=C([X])C(=O)OC1</smiles>

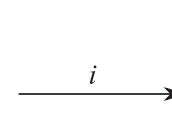

$\mathrm{X}=\mathrm{Br} 14$

$\mathrm{X}=\mathrm{Cl} 15$
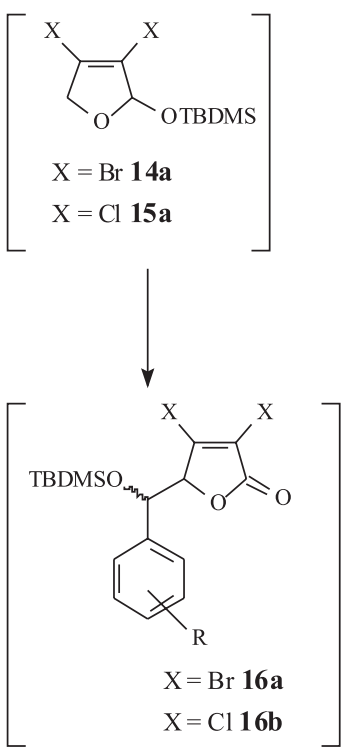

17: $\mathrm{X}=\mathrm{Br}, \mathrm{R}=\mathrm{H}(15 \%)$

18: $X=B r, R=3,4-$ dioxametil $(65 \%)$

19: $\mathrm{X}=\mathrm{Br}, \mathrm{R}=4-\mathrm{NO}_{2}(13 \%)$

20: $\mathrm{X}=\mathrm{Cl}, \mathrm{R}=3$,4-dioxametil $(70 \%)$

21: $\mathrm{X}=\mathrm{Cl}, \mathrm{R}=4-\mathrm{NO}_{2}(10 \%)$

Esquema 1. Metodologia para a síntese de 3,4-di-halo-5-alquilidenofuran2(5H)-onas (17 a 21). i) ArCHO, TBDMSOTf, DIPEA, $\mathrm{CH}_{2} \mathrm{Cl}_{2}$, t. a. 1 h, ii) $D B U$, refluxo $1 \mathrm{~h}$

xicos, catalisadores de paládio e de Wilkinson de custos elevados, e também requer longo tempo de reação (cerca de 5 dias). Em função desses aspectos, esta etapa do trabalho foi voltada para o desenvolvimento de outro procedimento experimental mais adequado para a introdução do grupo isopropila na posição 4 do anel lactônico. Nesse sentido optou-se pela investigação de condições experimentais envolvendo adição de Michael de di-isopropilcuprato de lítio no composto $\mathbf{1 4}$ com eliminação de brometo (Esquema 2). As condições experimentais avaliadas e os rendimentos dos produtos são apresentados na Tabela 1 .

Inicialmente a reação de adição do grupo isopropila foi realizada utilizando-se 1 equivalente de $\mathrm{CuI}$ na ausência de ácido de Lewis como catalisador, em THF e a $-78^{\circ} \mathrm{C}$ (Tabela 1 , experimento 1$)$. Nessas condições, o produto desejado (22) foi formado em apenas $2 \%$ de<smiles>O=C1OCC(Br)=C1Br</smiles>

14

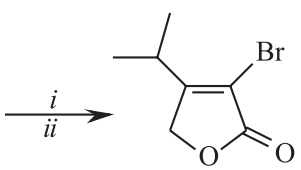

22
Esquema 2. Metodologia para a síntese de 3-bromo-4-isopropilfuran-2(5H)ona (22). i) i- $\mathrm{PrMgBr}$ (4 eq.), CuI (2 eq.), t.a. $40 \mathrm{~min}$; ii) $\mathrm{Me}_{3} \mathrm{SiCl}$ (1 eq.), $\mathrm{Et}_{2} \mathrm{O} / \mathrm{THF}$ (1:3), $-78^{\circ} \mathrm{C}, 1$ h, t.a. $15 \mathrm{~h}$

rendimento. $\mathrm{O}$ uso de $\mathrm{CuI}$ na presença de clorotrimetilsilano (Tabela 1, experimento 2) resultou em considerável melhoria do rendimento do produto 22 (63\%). O uso de $\mathrm{BF}_{3}$ em substituição ao clorotrimetilsilano forneceu apenas $48 \%$ do produto (Tabela 1, experimento 3). Diversos experimentos variando a fonte de cobre $(\mathrm{CuI}$ ou $\mathrm{CuBr}$. $\mathrm{Me}_{2} \mathrm{~S}$ ), solvente (éter dietílico ou THF), ácido de Lewis $\left(\mathrm{Me}_{3} \mathrm{SiCl}\right.$ ou $\left.\mathrm{BF}_{3} \cdot \mathrm{Et}_{2} \mathrm{O}\right)$ e temperatura $\left(0 \mathrm{ou}-78^{\circ} \mathrm{C}\right)$ foram realizados (Tabela 1$)$.

De acordo com os resultados da Tabela 1 , o melhor rendimento (93\%) foi alcançado no experimento 6, utilizando-se $\mathrm{CuBr} . \mathrm{Me}_{2} \mathrm{~S}$ (1 eq.) e $\mathrm{BF}_{3}$ ( 2 eq.). $\mathrm{O}$ uso de menor quantidade do ácido de Lewis resultou em redução no rendimento do produto (55\%, experimento 7). A redução na quantidade de $\mathrm{CuBr} . \mathrm{Me}_{2} \mathrm{~S}(0,5$ eq.) resultou ainda em um maior decréscimo no rendimento ( $28 \%$, experimento 8 ).

$\mathrm{O}$ uso de éter dietílico como solvente, mesmo se realizando as reações a $0{ }^{\circ} \mathrm{C}$, não resultou em melhoria do rendimento (experimentos 9 a 14).

Para a introdução do grupo benzila na posição 3 da lactona 22 vários experimentos foram realizados, todavia a metodologia que resultou no produto desejado envolveu o uso do acoplamento de $\mathrm{Ne}$ gishi, ${ }^{51}$ utilizando-se brometo de benzilzinco e tetrakis(trifenilfosfina) paládio (0) $\left[\mathrm{Pd}\left(\mathrm{Ph}_{3}\right)_{4}\right]$ (Esquema 3). A lactona 23 foi obtida com rendimento superior $(36 \%)$ ao descrito na literatura (25\%), quando também se utilizou o acoplamento de Negishi. ${ }^{27}$

Finalmente, a reação de alquilidenação entre a lactona 23 e aldeídos aromáticos substituídos forneceu os $\gamma$-alquilidenobutenolídeos 24 a 28 , análogos aos nostoclídeos, com rendimentos de 22 a $83 \%$ (Esquema 3). Conforme discutido anteriormente para a síntese de 3,4-di-halo-5alquilidenofuran-2 $(5 H)$-onas, também ocorreu a formação de isômeros com configuração $Z$ para a ligação dupla exocíclica, tendo em vista a presença do grupo isopropila na posição $\beta$ do anel lactônico.

Tabela 1. Condições para a otimização da metodologia alternativa para o preparo da lactona 22

\begin{tabular}{|c|c|c|c|c|c|c|c|c|}
\hline Experimento & $\mathrm{CuX}$ & $\begin{array}{c}\mathrm{CuX} \\
\text { (equiv.) }\end{array}$ & Ácido de Lewis & $\begin{array}{l}\text { Ácido de Lewis } \\
\text { (equiv.) }\end{array}$ & $\begin{array}{l}\text { Solvente } \\
\text { (anidro) }\end{array}$ & $\begin{array}{c}\text { Temperatura } \\
\left({ }^{\circ} \mathrm{C}\right)\end{array}$ & $\begin{array}{l}\text { Rendimento } \\
(\%) \text { de } 22\end{array}$ & $\begin{array}{c}\text { Rendimento } \\
\text { (\%) de recuperação de } \mathbf{1 4}\end{array}$ \\
\hline 1 & $\mathrm{CuI}$ & 1 & - & - & THF & -78 & 2 & - \\
\hline 2 & $\mathrm{CuI}$ & 1 & $\mathrm{Me}_{3} \mathrm{SiCl}$ & 2 & THF & -78 & 63 & 11 \\
\hline 3 & $\mathrm{CuI}$ & 1 & $\mathrm{BF}_{3} \cdot \mathrm{Et}_{2} \mathrm{O}$ & 2 & THF & -78 & 48 & 4 \\
\hline 4 & $\mathrm{CuBr} \cdot \mathrm{Me}_{2} \mathrm{~S}$ & 1 & - & - & THF & -78 & 11 & - \\
\hline 5 & $\mathrm{CuBr}_{\mathrm{Me}} \mathrm{S}$ & 1 & $\mathrm{Me}_{3} \mathrm{SiCl}$ & 2 & THF & -78 & 27 & - \\
\hline 6 & $\mathrm{CuBr}_{\mathrm{Me}} \mathrm{S}$ & 1 & $\mathrm{BF}_{3} \cdot \mathrm{Et}_{2} \mathrm{O}$ & 2 & THF & -78 & 93 & 1 \\
\hline 7 & $\mathrm{CuBr} \cdot \mathrm{Me}_{2} \mathrm{~S}$ & 1 & $\mathrm{BF}_{3} \cdot \mathrm{Et}_{2} \mathrm{O}$ & 1 & THF & -78 & 55 & - \\
\hline 8 & $\mathrm{CuBr}_{\mathrm{Me}} \mathrm{S}$ & 0,5 & $\mathrm{BF}_{3} \cdot \mathrm{Et}_{2} \mathrm{O}$ & 1 & THF & -78 & 28 & - \\
\hline 9 & $\mathrm{CuI}$ & 1 & _ & _- & $\mathrm{Et}_{2} \mathrm{O}$ & 0 & 4 & _ \\
\hline 10 & $\mathrm{CuI}$ & 1 & $\mathrm{Me}_{3} \mathrm{SiCl}$ & 2 & $\mathrm{Et}_{2} \mathrm{O}$ & 0 & 55 & 4 \\
\hline 11 & $\mathrm{CuI}$ & 1 & $\mathrm{BF}_{3} \cdot \mathrm{Et}_{2} \mathrm{O}$ & 2 & $\mathrm{Et}_{2} \mathrm{O}$ & 0 & 64 & 11 \\
\hline 12 & $\mathrm{CuBr} \cdot \mathrm{Me}_{2} \mathrm{~S}$ & 1 & - & - & $\mathrm{Et}_{2} \mathrm{O}$ & 0 & 19 & - \\
\hline 13 & $\mathrm{CuBr}_{\mathrm{Me}} \mathrm{S}$ & 1 & $\mathrm{Me}_{3} \mathrm{SiCl}$ & 2 & $\mathrm{Et}_{2} \mathrm{O}$ & 0 & 39 & 26 \\
\hline 14 & $\mathrm{CuBr}_{\mathrm{Me}} \mathrm{S}$ & 1 & $\mathrm{BF}_{3} \cdot \mathrm{Et}_{2} \mathrm{O}$ & 2 & $\mathrm{Et}_{2} \mathrm{O}$ & 0 & 22 & 78 \\
\hline
\end{tabular}




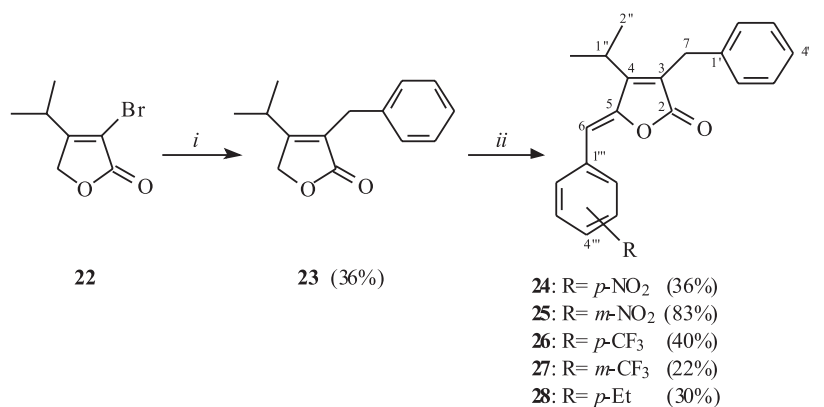

Esquema 3. Procedimentos para a síntese dos compostos 24 a 28. i) $\mathrm{C}_{6} \mathrm{H}_{6} \mathrm{CH}_{2} \mathrm{ZnBr}$ (2 eq.), $\mathrm{Pd}\left(\mathrm{PPh}_{3}\right)_{4}(5 \mathrm{~mol} \%), \mathrm{DMF}: \mathrm{THF}(1: 1), 60^{\circ} \mathrm{C}, 5 \mathrm{~h}$, $36 \%$; ii) $\mathrm{C}_{6} \mathrm{H}_{5} \mathrm{CHO}$, DIPEA, TBDMSOTf

\section{Avalliação da atividade antimicrobiana de furanonas halogenadas e de compostos análogos aos nostoclídeos}

Considerando a grande demanda para a busca de novas substâncias com atividade antimicrobiana, foi realizada a avaliação in vitro dos compostos 9 a $\mathbf{1 5}$ e $\mathbf{1 7}$ a $\mathbf{2 8}$, utilizando-se o teste de difusão em Ágar. ${ }^{41,52}$ Trata-se de um método padronizado pelo NCCLS (National Committee for Clinical Laboratory Standards) que permite classificar a bactéria como susceptível (S), intermediária (I) ou resistente (R) ao agente antimicrobiano.

Foram selecionadas cepas das bactérias Gram-positivas Staphylococcus aureus, Bacillus cereus e Gram-negativa Escherichia coli, por apresentarem elevada resistência a vários antibióticos de uso clínico.

A Tabela 2 apresenta os valores do halo de inibição dos compostos 9 a 15 e 17 a 28 , após 48 h de incubação.

O efeito inibitório dos compostos 9 a $\mathbf{1 3}$ e $\mathbf{2 4}$ a $\mathbf{2 8}$ sobre as bactérias foi pouco pronunciado, em comparação ao apresentado pelos antibióticos utilizados como controle. Foi demonstrado recentemente em estudo da correlação de estrutura química de diversos análogos aos nostoclídeos e atividade biológica $(\mathrm{QSAR})^{53}$ que a baixa atividade fitotóxica observada para alguns dos compostos avaliados pode estar correlacionada à baixa solubilidade dos mesmos em água. Embora os compostos tenham sido inicialmente solubilizados em DMSO devido à pouca solubilidade em água, a baixa atividade antimicrobiana pode ser devida em parte à baixa difusão desses compostos no meio aquoso em que os testes foram realizados.

A presença de átomos de halogênios nas posições 3 e 4 do anel lactônico parece potencializar a atividade antimicrobiana. As lactonas 14 e $\mathbf{1 5}$ mostraram efeito inibitório comparável ao dos antibióticos gentamicina e estreptomicina. Dos compostos pertencentes à classe das 3,4-di-halo-5-arilidenofuran-2(5H)-onas (17 a 21), a lactona $\mathbf{1 7}$ foi a que apresentou maior atividade, pois inibiu o crescimento de $S$. aureus e $B$. cereus de forma semelhante aos antibióticos estreptomicina e eritromicina. Em relação à bactéria $B$. cereus, observou-se que a lactona $\mathbf{2 0}$ apresentou atividade similar à do antibiótico penicilina, e que as lactonas 18, 19 e 21 causaram halo de inibição superior em relação ao mesmo antibiótico. Vale ressaltar que devido à pouca disponibilidade de material, a lactona 21 foi utilizada em menor quantidade $(100 \mu \mathrm{g} / \mathrm{disco})$ em relação aos demais compostos $(200 \mu \mathrm{g} / \mathrm{disco})$ e, ainda assim, causou inibição próxima à do antibiótico vancomicina.

A atividade antimicrobiana de lactonas halogenadas tem sido descrita na literatura. ${ }^{9} \mathrm{O}$ estudo de modelagem molecular para avaliar o efeito inibitório de derivados $\mathrm{N}$-acil-3-aminofuran-2(5H)onas halogenados e não halogenados sobre a síntese de proteínas em bactérias Gram-negativas mostrou que os compostos halogenados são mais ativos, devido à interação específica com proteínas da família LuxR..$^{54}$
Tabela 2. Valores dos halos de inibição dos compostos 9 a 15 e 17 a 28, após $48 \mathrm{~h}$, na concentração de $200 \mu \mathrm{g} / \mathrm{disco}$

Diâmetro da zona de inibição (mm)

\begin{tabular}{|c|c|c|c|}
\hline \multirow[t]{2}{*}{ Micro-organismos } & \multirow{2}{*}{$\begin{array}{c}\mathrm{G}(-) \\
\text { E. coli }\end{array}$} & \multicolumn{2}{|c|}{$\mathrm{G}(+)$} \\
\hline & & S. aureus & B. cereus \\
\hline \multicolumn{4}{|l|}{ Compostos } \\
\hline 9 & $6 \mathrm{Ac}$ & $6 \mathrm{Ag}$ & $6 \mathrm{Ah}$ \\
\hline 10 & $6 \mathrm{Ac}$ & $6 \mathrm{Ag}$ & $6 \mathrm{Ah}$ \\
\hline 11 & $6 \mathrm{Ac}$ & $6 \mathrm{Ag}$ & $6 \mathrm{Ah}$ \\
\hline 12 & $6 \mathrm{Ac}$ & $6 \mathrm{Ag}$ & $6 \mathrm{Ah}$ \\
\hline 13 & $6 \mathrm{Ac}$ & $6 \mathrm{Ag}$ & $6 \mathrm{Ah}$ \\
\hline 14 & $17 \mathrm{Ba}$ & $7 \mathrm{Cfg}$ & $20 \mathrm{Ab}$ \\
\hline 15 & $16 \mathrm{Aab}$ & $7 \mathrm{Bfg}$ & 16 Ade \\
\hline 17 & $8 \mathrm{Bc}$ & $15 \mathrm{Ad}$ & 17 Acd \\
\hline 18 & $6 \mathrm{Bc}$ & $10 \mathrm{Ae}$ & $10 \mathrm{Afg}$ \\
\hline 19 & $7 \mathrm{Bc}$ & $10 \mathrm{Ae}$ & $10 \mathrm{Afg}$ \\
\hline 20 & $6 \mathrm{Bc}$ & 9 Abef & $7 \mathrm{Bh}$ \\
\hline $21 *$ & $6 \mathrm{Bc}$ & $6 \mathrm{Bg}$ & $11 \mathrm{Afg}$ \\
\hline 22 & $6 \mathrm{Ac}$ & 8 Aefg & $8 \mathrm{Agh}$ \\
\hline 23 & $6 \mathrm{Ac}$ & 8 Aefg & $6 \mathrm{Ah}$ \\
\hline 24 & $6 \mathrm{Ac}$ & $6 \mathrm{Ag}$ & $6 \mathrm{Ah}$ \\
\hline 25 & $6 \mathrm{Ac}$ & $6 \mathrm{Ag}$ & $6 \mathrm{Ah}$ \\
\hline 26 & $6 \mathrm{Ac}$ & $6 \mathrm{Ag}$ & $6 \mathrm{Ah}$ \\
\hline 27 & $6 \mathrm{Ac}$ & $6 \mathrm{Ag}$ & $6 \mathrm{Ah}$ \\
\hline \multirow[t]{2}{*}{28} & $6 \mathrm{Ac}$ & $6 \mathrm{Ag}$ & $6 \mathrm{Ah}$ \\
\hline & Controle & & \\
\hline Vancomicina $30 \mu \mathrm{g}$ & $13 \mathrm{Bb}$ & $24 \mathrm{Ac}$ & $13 \mathrm{Bb}$ \\
\hline Penicilina G 10 UI & $8 \mathrm{Bc}^{*}$ & $51 \mathrm{Aa}$ & $8 \mathrm{Bgh}$ \\
\hline Eritromicina $15 \mu \mathrm{g}$ & $6 \mathrm{Cc}^{*}$ & $31 \mathrm{Ab}$ & $18 \mathrm{Bbcd}$ \\
\hline Gentamicina $10 \mu \mathrm{g}$ & $16 \mathrm{Ba}$ & $18 \mathrm{ABd}$ & $20 \mathrm{Abc}$ \\
\hline Estreptomicina $10 \mu \mathrm{g}$ & $16 \mathrm{Ba}$ & $15 \mathrm{Bd}$ & $24 \mathrm{Aa}$ \\
\hline Água estéril & $6 \mathrm{Ac}$ & $6 \mathrm{Ag}$ & $6 \mathrm{Ah}$ \\
\hline DMSO & $6 \mathrm{Ac}$ & $6 \mathrm{Ag}$ & $6 \mathrm{Ah}$ \\
\hline
\end{tabular}

*Utilizado na concentração de $100 \mu \mathrm{g} /$ disco. Médias seguidas da mesma letra, maiúsculas na horizontal, e minúsculas na vertical, não diferem entre si pelo teste de Tukey $(\mathrm{P} \leq 0,05)$, *bactérias resistentes.

\section{CONCLUSÃO}

A lactona 23, um intermediário na síntese de nostoclídeos, foi preparada por uma nova metodologia com rendimentos superiores aos relatados na literatura. As atividades antimicrobianas de furanonas halogenadas (17 a 22) e dos análogos aos nostoclídeos 9 a $\mathbf{1 3}$ e 24 a 28 foram avaliadas contra as bactérias $S$. aureus, B. cereus e $E$. coli. De modo geral, as lactonas apresentaram atividade moderada contras as bactérias avaliadas, mas em alguns casos foi observada atividade comparável aos antibióticos utilizados como controle. Comparando-se as atividades antimicrobianas dos compostos 9 a $\mathbf{1 3}$ com os compostos contendo o grupo isopropila na posição 4 do anel lactona ( 24 a $\mathbf{2 8}$ ), não se observou diferença significativa entre as atividades, demonstrando a princípio que o grupo isopropila parece não ser essencial para a atividade sobre as bactérias avaliadas. De modo geral, os compostos halogenados foram os mais ativos, e os 
resultados obtidos nesst trabalho com as lactonas halogenadas são motivadores para o avanço na síntese de novos derivados e continuidade nos estudos para a busca de substâncias de ação antimicrobiana.

\section{AGRADECIMENTOS}

Ao Conselho Nacional de Desenvolvimento Científico e Tecnológico (CNPq) pelas bolsas de pesquisas (A. J. Demuner, C. R. A. Maltha, L. C. A. Barbosa) e à Fundação de Amparo à Pesquisa do Estado de Minas Gerais (FAPEMIG) pelo apoio financeiro.

\section{REFERÊNCIAS}

1. Mantilla, S. P. S. R.; Franco M.; Oliveira, L. A. T.; Santos, E. B.; Gouvêa, R.; Braz. J. Vet. Res. Anim. Sci. 2008, 45, 116.

2. Montelli, A. C.; Sadatsune, T.; Antibioticoterapia para o clínico, Sociedade Brasileira de Microbiologia: Rio de Janeiro, 2001, p. 7-53.

3. Aureli, P.; Ferrini, A. M.; Mannoni, V.; Hodzic, S.; Wedell-Weergaard, C.; Oliva, B.; Int. J. Food Microbiol. 2003, 83, 325.

4. Wunder, R. S.; Arq. Bras. Med. Naval 2004, 65, 7.

5. Sjögren, A. L.; Johnson, E.; Hedner, M.; Dahlström, U.; Göransson, H.; Shirani, J.; Bergman, P. R.; Jonsson, L.; Peptides 2006, 27, 2058.

6. Caine, D.; Ukachukwu, V. C.; J. Org. Chem. 1985, 50, 2195.

7. Manefield, M.; Rasmussen, T. B.; Henzter, M.; Andersen, J. B.; Steinberg, P.; Kjelleberg, S.; Givskov, M.; Microbiology 2002, 148, 1119.

8. Baveja, J. K.; Willcox, M. D. P.; Hume, E. B. H.; Kumar, N.; Odell, R.; Warren, L. A. P.; Biomaterials 2004, 25, 5003.

9. Baveja, J. K.; Li, G.; Nordon, R. E.; Hume, E. B. H.; Kumar, N.; Willcox, M. D. P.; Warren, L. A. P.; Biomaterials 2004, 25, 5013.

10. Bataineh, S. A. A.; Britcher, L. G.; Griesser, H. J.; Surf. Sci. 2006, 600, 952.

11. Han, Y.; Hou, S.; Simon, K. A.; Ren, D.; Bioorg. Med. Chem. Lett. 2008, 18,1006 .

12. Rice, S. A.; McDouglad, D.; Kumar, N.; Kjelleberg, S.; Curr. Opin. Investig. Drugs 2005, 6, 178.

13. Souza, M. V. N.; Mini-Rev. Org. Chem. 2005, 2, 139

14. Nys, R.; Wright, A. D.; Konig, G. M.; Sticher, O.; Tetrahedron 1993, 49, 11213.

15. Beechan, C. M..; Sims, J. J.; Tetrahedron Lett. 1979, 35, 1649.

16. Janssens, J. C. A.; Steenackers, H.; Robijns, S.; Gellens, E.; Levin, J.; Zhao, H.; Hermans, K.; De Coster, D.; Verhoeven, T. L.; Marchal, K.; Vanderleyden, J.; De Vos, D. E.; De Keersmaecker, S. C. J.; Appl. Environ. Microbiol. 2008, 74, 6639.

17. Kjelleberg, S.; Steinberg, P. D.; Holmstrom C.; Back, A.; Patente $N^{o}$. PCT/AU99/00284, 1999.

18. Holmstrom, C.; Kjelleberg, S.; Patente No. PCT/AUO1/00295, 2001.

19. Baveja, J. K.; Willcox, M. D. P.; Hume, E. B. H.; Kumar, N.; Odell, R.; Warren, L. A. P.; Biomaterials 2004, 25, 5003.

20. Zhang, R.; Chan, D.; Jessica, S.; Iskander, G.; Black, D. S.; Kumar, N.; ARKIVOC 2009, 102.

21. Baer, H.; Holden; Seegal, B. C.; J. Biol. Chem. 1946, 162, 65.

22. Miao, S.; Andersen, R. J.; J. Org. Chem. 1991, 56, 6275.

23. Davidson, B. S.; Ireland, C. M.; J. Nat. Prod. 1990, 53, 1036.

24. Antane, C. E.; Caufield, W.; Hu, D.; Keeney, P.; Labthavikul, P.; Morris, V; Naughton, S. M.; Petersen, P. J.; Rasmussen, B. A.; Singh, G.; Yang, Y.; Bioorg. Med. Chem. Lett. 2006, 16, 176.

25. Xuemin, Y.; Yuzuru, S.; Steiner, J. R.; Clardy, J.; Tetrahedron Lett. 1993, $34,761$.

26. Boukouvalas, J.; Maltais, F.; Lachance, N.; Tetrahedron Lett. 1994, 35 , 7897.

27. Bellina, F.; Rossi, R.; Synthesis 2002, 2729.

28. Kar, A.; Gogoi, S.; Argade, N. P.; Tetrahedron 2005, 61, 5297.

29. Barbosa, L. C. A.; Cutler, D.; Mann, J.; Crabbe, M. J.; Kirb, C.; Warhurst, D. C.; J. Chem. Soc., Perkin Trans. 1 1992, 3251.
30. Barbosa, L. C. A.; Cutler, D.; Mann, J.; Crabbe, M. J.; Kirb, C.; Warhurst, D. C.; J. Chem. Soc., Perkin Trans. 1 1996, 11, 1101.

31. Teixeira, R. R.; Barbosa, L. C. A.; Maltha, C. R. A.; Rocha, M. E.; Bezerra, D. P.; Lotufo, L. V. C.; Pessoa, C.; Moraes, M. O.; Molecules 2007, 12, 1101

32. Barbosa, L. C. A.; Kiran, Y. B.; Reddy, C. D.; Gunasekar, D.; Leon, A.; Eur. J. Med. Chem. 2008, 43, 885.

33. Carvalho, M. R.; Barbosa, L. C. A.; Queiroz, J. H.; Howarth, O. W.; Tetrahedron Lett. 2001, 42, 809.

34. Moreira, M. D.; Picanço, M. C.; Barbosa, L. C. A.; Guedes, R. N. C.; Barros, E. C.; Campos, M. R.; Pest. Manag. Sci. 2007, 63, 615.

35. Barbosa, L. C. A.; Barcelos, F. F.; Demuner, A. J.; Santos, M. A. D.; Nematropica 1999, 29, 81.

36. Demuner, A. J.; Barbosa, L. C. A.; Nascimento, J. C.; Vieira, J. J.; Santos, M. A.; Quim. Nova 2003, 26, 335.

37. Barbosa, L. C. A.; Ferreira, M. L.; Demuner, A. J.; Silva, A. A.; Pereira, R. C.; Quim. Nova 2001, 24, 751; Barbosa, L. C. A.; Maltha, C. R. A.; Borges, E. E. L.; Quim. Nova 2002, 25, 203; Barbosa, L. C. A.; Maltha, C. R. A.; Demuner, A. J.; Filomeno, C. A.; Silva, A. A.; Quim. Nova 2004, 27, 241; Barbosa, L. C. A.; Maltha, C. R. A.; Demuner, A. J.; Ganen, F. R.; Silva, A. A.; Quim. Nova 2005, 28, 444; Alvarenga, E. S.; Barbosa, L. C. A.; Saliba, W. A.; Arantes, F. F. P.; Demuner, A. J.; Silva, A. A.; Quim. Nova 2009, 32, 401; Barbosa, L. C. A ; Rocha, M. E.; Teixeira, R. R.; Maltha, C. R. A.; Forlani, G.; J. Agric. Food Chem. 2007, 55, 8562; Lima, L. S.; Barbosa, L. C. A.; Alvarenga, E. S.; Demuner, A. J.; Silva, A. A.; Austr. J. Chem. 2003, 36, 625; Demuner, A. J.; Barbosa, L. C. A.; Veiga, T. A. M.; Barreto. R. W.; King-Diaz, B.; Hennsen, B. L.; Biochem. System. Ecol. 2006, 34, 790.

38. Barbosa, L. C. A.; Demuner, A. J.; Alvarenga, E. S.; Oliveira, A.; KingDiaz, B.; Hennsen, B. L.; Pest. Manag. Sci. 2006, 62, 214.

39. Teixeira, R. R.; Barbosa, L. C. A.; Forlani, G.; Veloso, D. P.; Carneiro, J. W. M.; J. Agric. Food Chem. 2008, 56, 2321.

40. Barbosa, L. C. A.; Demuner, A. J.; Maltha, C. R. A.; Teixeira, R. R.; Souza, K. A. P.; Bicalho, K. U. S.; Z. Naturforsch., B: J. Chem. Sci. 2009, 64, 245.

41. Sejas, L. M.; Silbert; A. O. R.; Sader, H. S.; J. Bras. Patol. Med. Laboratorial 2003, 39, 27.

42. Perrin, D. D.; Armarego, W. L. F.; Purification of Laboratory Chemicals, $3^{\text {rd }}$ ed.; Pergamon: Oxford, 1988

43. Bellina, F.; Anselmi, C.; Stephane, V.; Mannina, L.; Rossi, R.; Tetrahedron 2001, 57, 9997.

44. Bellina, F.; Anselmi, C.; Martina, F.; Rossi, R.; Eur. J. Org. Chem. 2003, 2290.

45. Salustiano, V. C.; Andrade, N. J.; Soares, N. F. F.; Lima, J. C.; Bernardes, P. C.; Fernandes, P. E.; Food Control 2009, 20, 439.

46. NCCLS; Performance Standards for Antimicrobial Disk Susceptibility Tests; Approved Standard $-8^{\text {th }}$ ed.; NCCLS document M2-A8 [ISBN 1-56238-485-6]. NCCLS: Wayne, 2003.

47. Boukouvalas, J.; Beltran, P. P.; Lachance, N.; Cote, S.; Maltais, F.; Pouliot, M. A.; Synlett 2007, 219.

48. Sarma, K. P.; Zhang, J.; Curran, T. T.; J. Org. Chem. 2007, 72, 3311.

49. Teixeira, R. R.; Barbosa, L. C. A.; Varejão, J. S. O.; Pilo-Veloso, D.; Ellena, J.; Dorigueto, A. C.; Drew, M. G. B.; Ismail, F. M. D.; J. Mol. Struct. 2007, 837, 197.

50. Teixeira, R. R.; Barbosa, L. C. A.; Carneiro, J. W. M.; Correa, R. S.; Ellena, J.; Dorigueto, A. C.; J. Mol. Struct. 2009, 917, 1.

51. Negishi, E.; Anastasia, L.; Chem. Rev. 2003, 103, 1979.

52. Silva, C. J.; Barbosa, L. C. A.; Demuner, A. J.; Montanari, R. M.; Pinheiro, A. L.; Dias, I.; Andrade, N. J.; Quim. Nova 2010, 33, 104.

53. Teixeira, R. R.; Pinheiro, P. F.; Barbosa, L. C. A.; Carneiro, J. W. M.; Forlani, G.; Pest.. Manag. Sci. 2010, 66, 196.

54. Estephane, J.; Dauvergne, J.; Soulère, L.; Reverchon, S.; Bioorg. Med. Chem. Lett. 2008, 18, 4321. 